\title{
Stress Analysis of Hip and Knee Prostheses Using a Novel Biomaterial PTFE Glass Composite
}

\author{
Shankar S. Das*, Prasun Chakraborti \\ Department of Mechanical Engineering, National Institute of Technology, Agartala, Tripura, Pincode 799046, India
}

Corresponding Author Email: shankarswarup@gmail.com

https://doi.org/10.18280/ama_c.740104

Received: 29 December 2018

Accepted: 7 March 2019

\section{Keywords:}

hip and knee joint prostheses, acetabular cup, tibial liner, femoral ball, finite element modeling, PTFE glass composite

\begin{abstract}
Modern hip and knee prostheses are mostly available in Metal-on-Metal, Metal-on-Plastic, and Metal-on-Ceramic combinations. Use of Metal-on-Metal prostheses is restricted due to adverse effect of worn metallic particles in the body. Hence the manufacturers are focusing on the Metal-on-Plastic (MoP) prostheses for safe and reliable arthroplasty. Finite Element Modeling plays a great role in this direction for the design of high quality hip and knee prostheses. This study investigates the stress analysis and contact behavior of MoP hip and knee prostheses using a novel biomaterial called Poly-tetra Fluoroethylene with $25 \%$ glass composite coded as PTFE-25 for the acetabular and tibial liners by using ANSYS.16 software under various loads. The analysis revealed that the von-mises equivalent stress, and contact pressures for both the prostheses are highest in case of PTFE-25 as compared to that of the Ultra-high Molecular Weight Polyethylene (UHMWPE) articulating againest the Co-Cr components. Moreover, the total deformation in case of PTFE-25 is lesser as compared to UHMWPE under identical loading conditions. The higher the contact pressure the greater will be the wear resistance of the liners. This shows that PTFE-25 is a better candidate for the acetabular and tibial liners of the hip and knee prostheses.
\end{abstract}

\section{INTRODUCTION}

The hip and knee joints are the two major load carrying joints on the human body, available in shapes of spherical ball and socket and hinge joints respectively. The hip joint connects the femur and acetabulum, whereas the knee joint connects the femur and tibia of the of human body. The soul function of these joints is to sustain whole weight of the body in both static and dynamic postures [1]. Wear of the hip and knee liner surface is a serious drawback of total joint replacement (TJR) surgery. The aseptic profile loosening from the ultrahigh molecular weight polyethylene (UHMWPE) wear particles is the root cause of failure for metal-onpolyethylene (MoP) hip and knee prostheses [2]. Wear debris formed at the articulating face causes the prosthetic osteolysis, degradation of bone surrounding the prosthesis, which tends to a loss of implant anchoring in-vivo resulting fixation loss and severe thigh pain etc. In the case of a metal-onpolyethylene combination it is assumed that around 70 to $95 \%$ of wear debris in the osteolytic tissues is UHMWPE particles from the articulating surfaces [3]. In such cases, the femoral and tibial heads of hip and knee joints are replaced with a metallic heads supported with metal stem which is anchored into the hollow cavity inside the femoral bone with bone cement (PMMA) and the worn out components are replaced by a man-made polyethylene components [4]. In order to estimate the functioning life of hip and knee joint prostheses, it is necessary to recognize the modes of failure of these prostheses [5].

The total number of revision hip and knee arthroplasties is 48,273 and 38,559 respectively as per Finnish Arthroplasty Register-2016. Also the number of hip and knee arthroplasties exceeds 100,000 every year in United States of America as per their report [6]. These statistics shows that the demand for hip and knee arthroplasties has increased greatly during the last twenty years around the world. The number is expected to be increasing to a good figure in the forthcoming years. Principally, the failure of both hip and knee joint prostheses depend upon the sliding wear of the articulating interfaces of the joints. Many literatures enlightened the fatigue failure and wear of the prostheses in different ways [7].

Some of these reports also suggested that the fatigue fracture and fretting wear as common failure phenomena in case of the metal-on-metal (MoM) hip and knee prostheses [8]. Smart tools like hip and knee simulators can be employed for wear estimation hip and knee joint prostheses for different combination of materials. Such experimentation may be considered as a pre-clinical test for predicting the damage probability of the prostheses with respect to time as a remedial measure though it's not easy to create an anatomical system for dynamic analysis [9].

Individual testing of the components of hip and knee joint prostheses can be attained only by altering or modifying some conditions. Although the Total Hip and Knee Replacement operations are very successful, the problems or pain during motion still remain a challenge for some patients. This might be explained by surgical errors or by excessive deviations from the standard hip and knee anatomy which can lead to a different biomechanical behavior than what the prosthesis was designed by Al-Dirini et al. [10]. Most THR and TKRs function as surface replacements within the soft tissue envelope that surrounds the hip and knee. Consequently, positioning and sizing of the components will largely affect the post-operative result. Any lose guide wrong sizing will affect loads on the interface and tension in the ligaments. This will lead to deviation in knee mechanics inducing stiffness, 
instability and early loosening [11].

This study presents stress analysis of improved CAD models of hip and knee prostheses by using ANSYS.16. Separate analyses are made for total deformation as well as contact pressure using different combination of materials such as Co-Cr on UHMWPE, and Co-Cr on PTFE-25 etc. Generally, pure PTFE components are not used due to severe wear rate as per the statements of John Charnley after his first experiment on Metal-on-Plastic hip joint prosthesis. Therefore, an attempt has been made in this study to use a novel bio-material PTFE with $25 \%$ glass composite (PTFE-25) for the acetabular and femur distal liners of hip and knee joints under different loads. Comparative study has also been made to check the performance of both the PTFE-25 and UHMWPE components for hip and knee prostheses.

\section{MATERIALS AND METHODS}

The materials used for hip and knee prostheses should be highly biocompatible and the components should be manufactured only after satisfactory performances of the finite element model of the hip prosthesis and its numerical simulations. This section covers the properties of commonly used materials and the finite element modeling and simulation of a hip prosthesis.

\subsection{Materials used}

Various types of biomaterials have been developed in the recent past for manufacturing the hip prostheses [12]. In this study, we have considered one of the most commonly used biomaterial Cobalt-Chromium-Molybdenum (Co-Cr-Mo) alloy for femoral head and distal end of hip and knee prostheses. Similarly, the bio-plastic materials such as Ultrahigh Molecular Weight Polyethylene (UHMWPE) and Polytetra Fluroethylene with $25 \%$ glass composite (PTFE-25) as acetabular and tibial liners of the hip and knee prostheses. The properties of these biomaterials to be used for this analysis are listed in table-1 as follows:

Table 1. Material properties used for model analysis [9-12]

\begin{tabular}{|c|c|c|c|c|c|c|}
\hline 苞 & 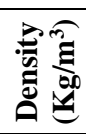 & 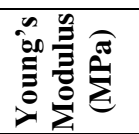 & 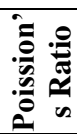 & 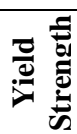 & 营 & 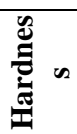 \\
\hline $\begin{array}{c}\mathrm{Co}-\mathrm{Cr}- \\
\mathrm{Al}\end{array}$ & 8300 & $2.30 \mathrm{E}+05$ & 0.3 & 612 & 970 & $\begin{array}{l}\mathrm{HB} \\
341\end{array}$ \\
\hline $\begin{array}{c}\text { UHMW } \\
\text { PE }\end{array}$ & 930 & $6.9 \mathrm{E}+02$ & 0.46 & 21 & 40 & D 68 \\
\hline PTFE & 2160 & $4.82 \mathrm{E}+02$ & 0.45 & 27 & 45 & D 50 \\
\hline PTFE-25 & 2250 & $\begin{array}{c}7.58 \\
E+02\end{array}$ & 0.48 & 15 & 27 & D 70 \\
\hline
\end{tabular}

\subsection{Finite element modeling and simulation}

The three dimensional geometric modeling of hip and knee joint prostheses plays a significant role during the performance study while in operation [13]. Therefore, an attempt has been made in this study to design and develop three basic components of a hip and knee prostheses such as femoral, acetabular, and tibial components by considering standard dimensions as per ISO-14242. These parts are properly designed one by one using SOLIDWORKS.16 software and then assembled together to form the total hip and knee prostheses and saved in. STEP format. Then both the hip and knee joint models indicating all parts as shown in Figures 1(a) \& (b) was imported in to the platform of ANSYS.16 for further processing in a high speed computer with Xeon processor at the CFD Lab of NIT Agartala. Prior to meshing of the models, the material properties are assigned to all the components of hip and knee prosthesis as listed in Table 1.

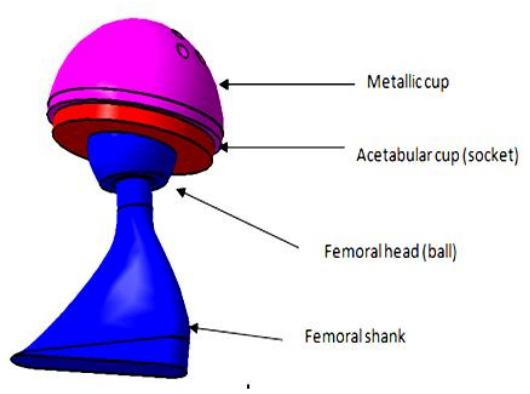

(a) Hip Joint Model

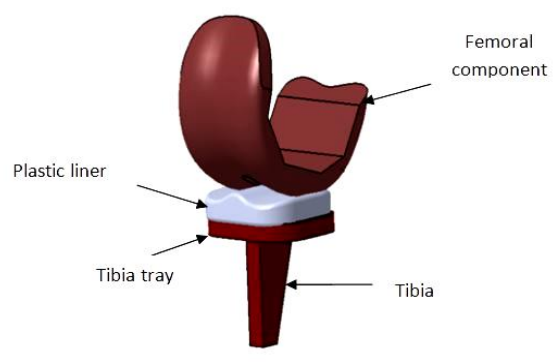

(b) Knee Joint Model

Figure 1. CAD model of hip and knee joint prostheses

In this simulation, we have considered the Co-Cr alloy for the femoral components, whereas the polymers like UHMWPE, PTFE and PTFE-25 for the acetabular and tibial components of hip and knee prostheses. Thereafter, tetrahedron meshing was generated for all the components of the prostheses with fine mesh of 10 nodes. The total number of elements for hip prosthesis is 31454 with 53467 nodes with mesh matrix aspect ratio varying from 1.1907 to 48.071 whereas the average value is 2.4623 with a standard deviation of 2.3805. In order to estimate the contact pressure accurately, fine mesh with element size $8 \mathrm{~mm}$ and 0.2 minimum edge lengths was selected for contact region. Each node of tetrahedron element has three degrees of freedom having a transition ratio of 0.272 with five maximum layers and a growth rate of 1.2. Component wise element, node, material details are given in Table 2.

Table 2. Statistics of the number of elements and nodes for hip prosthesis

\begin{tabular}{ccccc}
\hline $\begin{array}{c}\text { Modeling } \\
\text { Particulars }\end{array}$ & $\begin{array}{c}\text { Acetabular } \\
\text { Cup }\end{array}$ & $\begin{array}{c}\text { Femoral } \\
\text { Ball }\end{array}$ & Cup & Shank \\
\hline Nodes & 7862 & 4120 & 37271 & 4214 \\
Elements & 4502 & 2379 & 22283 & 2290 \\
Mesh Metric & \multicolumn{4}{c}{ Aspect Ratio } \\
Min & 1.2575 & 1.2289 & 1.1907 & 1.211 \\
Max & 14.735 & 6.3167 & 31.027 & 48.071 \\
Average & 2.0826 & 2.0105 & 2.57001 & 2.2558 \\
Standard & 0.6902 & 0.5075 & 2.60451 & 2.0342 \\
Deviation & & & & \\
\hline
\end{tabular}


Similarly, the total number of elements for knee prosthesis is 12082 with 8909 nodes with mesh matrix aspect ratio varying from 1.1717 to 42.2171 whereas the average value is 2.2671 with a standard deviation of 2.1246 . For estimation for the contact pressure accurately, fine mesh with element size 8 $\mathrm{mm}$ and 0.2 minimum edge lengths has been selected for contact region. Each node of tetrahedron element has 3 degrees of freedom with a transition ratio of 0.264 with 5 maximum layers and a growth rate of 1.2. The details of element, node, and material for the components are given in Table 3.

Table 3. Statistics of the number of elements and nodes for knee prosthesis

\begin{tabular}{cccc}
\hline $\begin{array}{c}\text { Modeling } \\
\text { Particulars }\end{array}$ & $\begin{array}{c}\text { Femoral } \\
\text { Component }\end{array}$ & Knee Insert & Tibia Shank \\
\hline Nodes & 6199 & 1740 & 970 \\
Elements & 3383 & 5637 & 3062 \\
Mesh Metric & & Aspect Ratio & \\
Min & 1.235 & 1.1245 & 1.1717 \\
Max & 12.735 & 6.1247 & 42.2147 \\
Average & 2.0241 & 2.0145 & 2.2671 \\
Standard & 0.6242 & 0.5241 & 2.1246 \\
Deviation & & & \\
\hline
\end{tabular}

\section{RESULTS}

With a specific end goal to analyze the hip and knee joint prosthesis under static load condition, the femoral distal end was constrained in every one of the headings. A load of 1500 $\mathrm{N}$ was applied at 20 degree angle to the proximal territory of the immense trochanter to simulate the abductor muscle force. Static examination was completed utilizing normal human body weight ranging from 600 to $1500 \mathrm{~N}$ during normal walking activities. The static investigations were performed on an advanced computer work station with Intel-Xeon $4.40 \mathrm{GHz}$ processor, 6GB RAM with the given load conditions. The Von-Mises Equivalent stress of the hip and knee prostheses for different loads acting at different contact points are shown in Figures 2(a) \&(b), and 3(a) \&(b) respectively. It is observed from the above finite element analysis that the equivalent stress increases with the increase in load and the maximum value occurs at the acetabular cup whereas the minimum value occurs at the bottom of the femur head in case of a hip prosthesis.

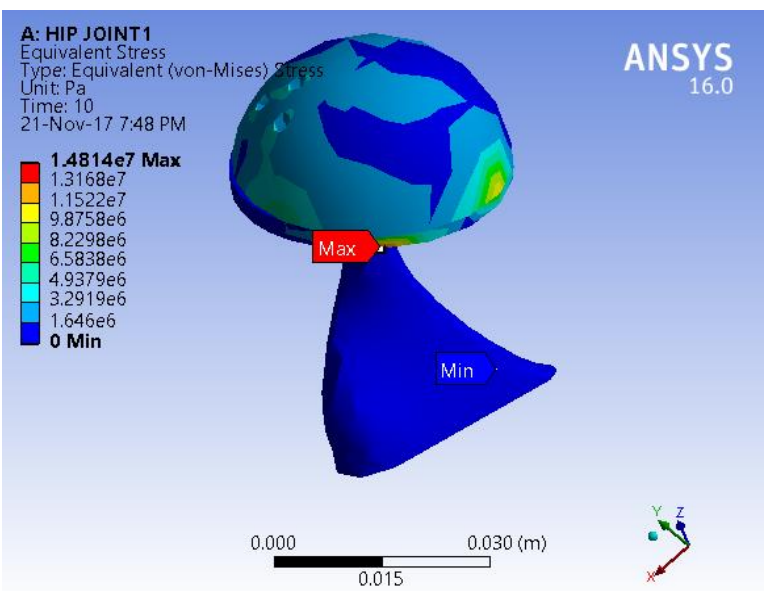

(a) For Co-Cr on UHMWPE Prosthesis

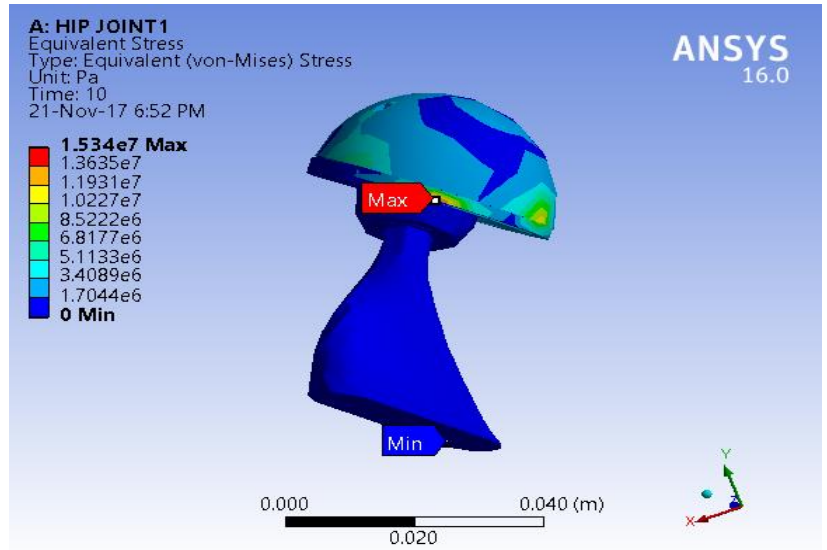

(b) For Co-Cr on PTFE-25 Prosthesis

Figure 2. Von-Mises eq stress for hip joint at $1500 \mathrm{~N}$

Similarly, the equivalent stress is maximum at distal end of femur indicated as red spots whereas the minimum indicated as blue color at the tibia of knee prosthesis. It is found from Figures 3 (a) \& (b) that the equivalent stress is $15.34 \mathrm{MPa}$ for PTFE-25 liner and 14.814 MPa for UHMWPE liner for hip and knee prostheses under a maximum load of $1500 \mathrm{~N}$. This shows that the hip and knee prostheses using PTFE-25 has higher strength than those using UHMWPE under similar loading conditions.

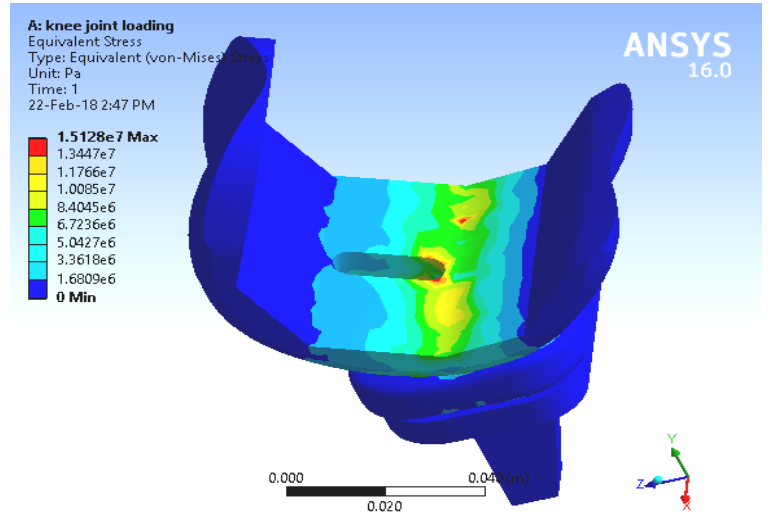

(a) For Co-Cr on UHMWPE Prosthesis

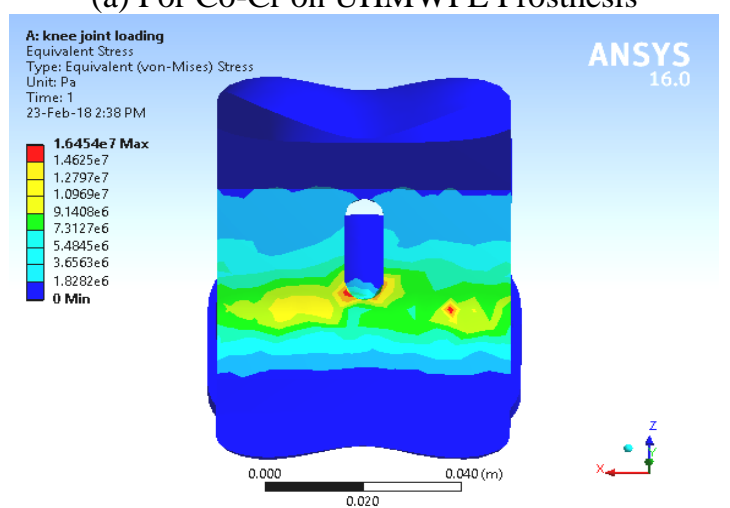

(b) For Co-Cr on PTFE-25 Prosthesis

Figure 3. Von-Mises eq stress for knee joint at $1500 \mathrm{~N}$

Further investigation from the plots of Von-mises equivalent stress versus load for hip and knee prostheses says that the stress is higher for PTFE-25 liners than the UHMWPE liners for all the applied loads ranging from 600 to $1500 \mathrm{~N}$ as shown in Figures 4 (a) \& (b) below. 


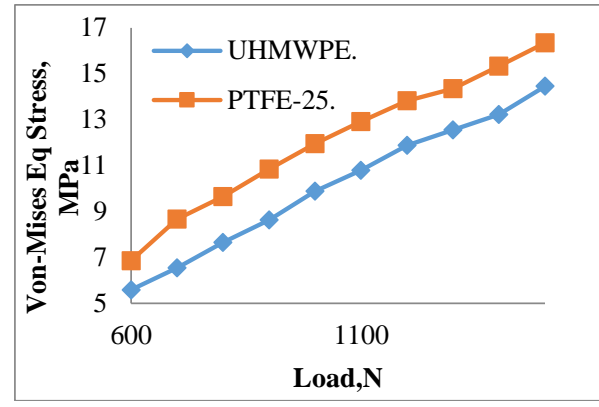

(a) For Hip Joint Prosthesis

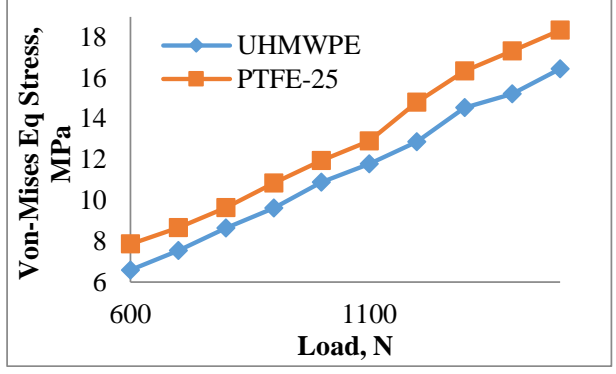

(b) For Knee Joint Prosthesis

Figure 4. Von-Mises equivalent stress Vs load

Everybody knows that the lower the total deformation the better will be the life of the hip and knee prostheses components. The total deformations maps of hip prostheses using for UHMWPE and HDHA-30 cups under load of 1500N are shown in Figures 5 (a) \& (b) respectively.

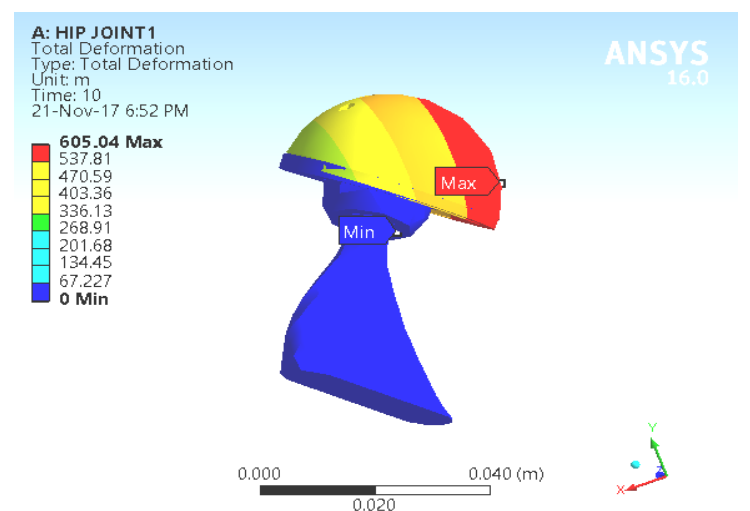

(a) For Co-Cr on UHMWPE Prosthesis

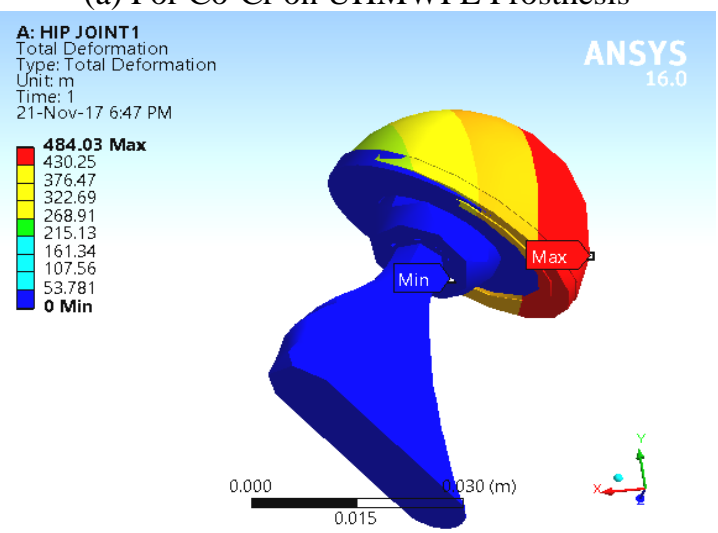

(b) For Co-Cr on PTFE-25 Prosthesis

Figure 5. Total deformation of Hip Joint at $1500 \mathrm{~N}$

Similarly, the total deformations maps of knee prostheses using UHMWPE and HDHA-30 inserts under load of 1500N are shown in Figures 6 (a) \& (b) respectively.

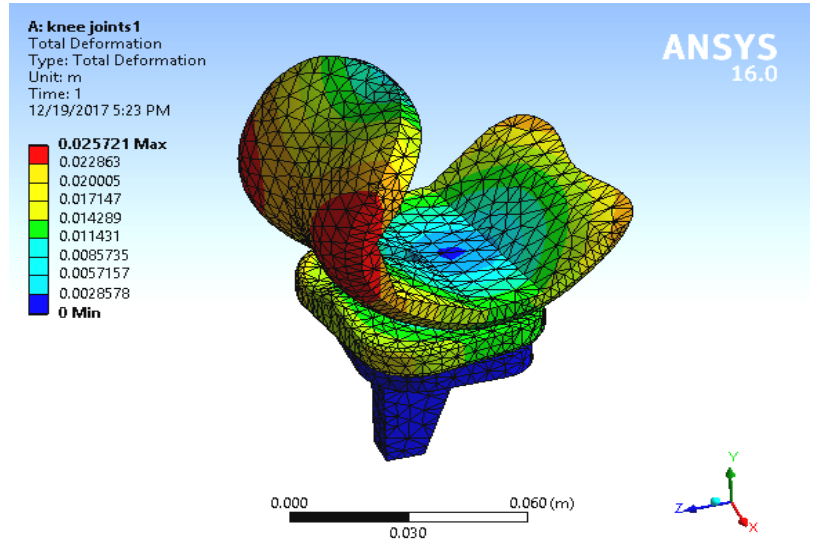

(a) For Co-Cr on UHMWPE Prosthesis

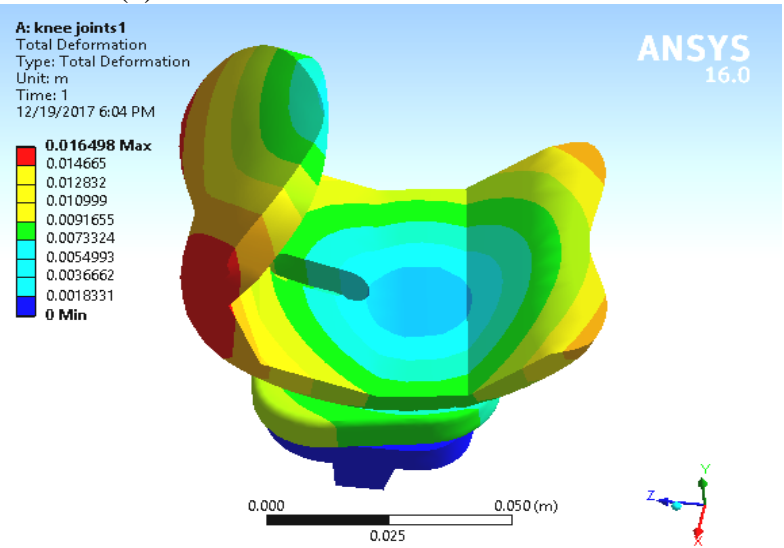

(b) For Co-Cr on PTFE-25 Prosthesis

Figure 6. Total deformation of knee joint at $1500 \mathrm{~N}$

It is found from the investigation that the total deformations in case of hip joint prostheses using PTFE-25 and UHMWPE liners are 484.03 and $605.04 \mathrm{~m}$ respectively under load of $1500 \mathrm{~N}$, whereas the similar figures for knee joint prostheses using PTFE-25 and UHMWPE are 0.016949 and $0.025721 \mathrm{~m}$ respectively under the same load. Similarly, the slopes of total deformation curves for PTFE-25 are found lower than that of UHMWPE under similar loads from Figures 7(a) \& (b).

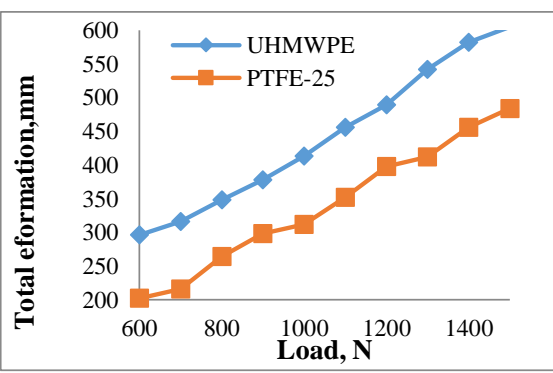

(a) For Hip Joint Prosthesis

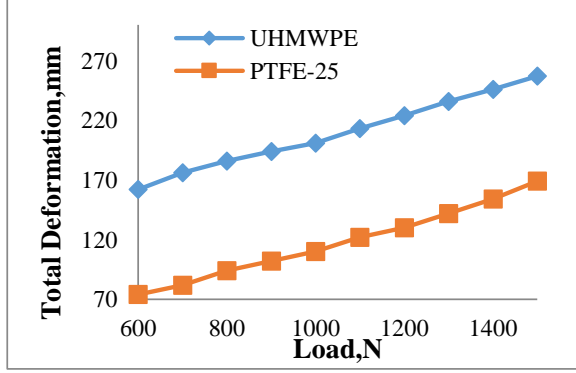

(b) For Knee Joint Prosthesis

Figure 7. Total deformation Vs load 
Though there is no significant change in the Von-Mises equivalent stress and total deformation of the various MoP prostheses, it is essential to investigate the contact pressure between the mating Metal and Plastic surfaces under the given load conditions.

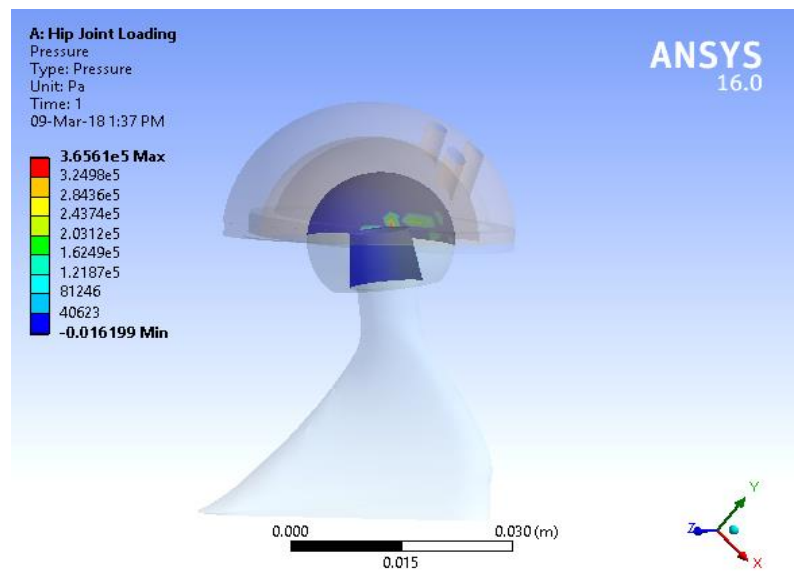

(a) For Co-Cr on UHMWPE Prosthesis

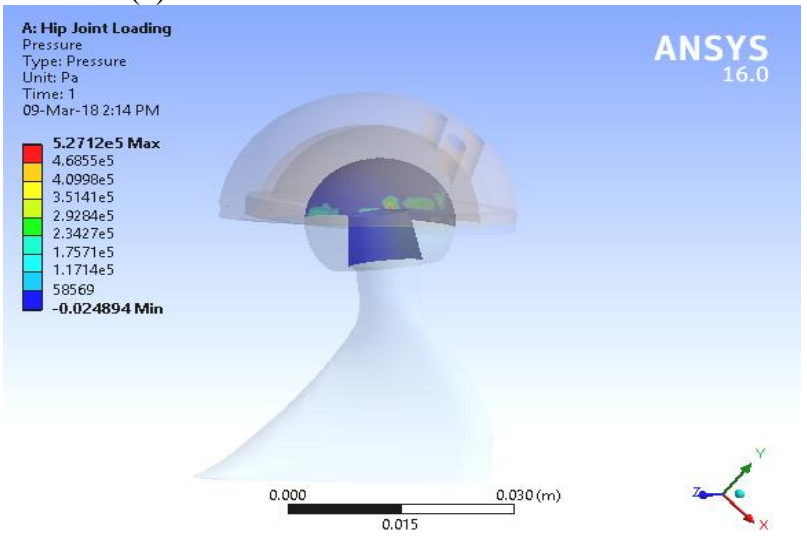

(a) For Co-Cr on PTFE-25 Prosthesis

Figure 8. Contact pressure of hip joint at $1500 \mathrm{~N}$

The contact pressures of PTFE-25 and UHMWPE components for hip prosthesis under a load of $1500 \mathrm{~N}$ are shown in Figures 8(a) \& (b) and the same for knee prosthesis are shown in Figures 9(a) \& (b), respectively.

The variation of contact pressure for different plastic surfaces mating with Co-Cr and UHMWPE and PTFE-25 are plotted separately in Figures 10(a) \& (b) for better comparison. It is found that the slope of the curve for PTFE-25 component is higher than that of the UHMWPE for both hip and knee prostheses for a series of applied loads from 600 to $1500 \mathrm{~N}$.

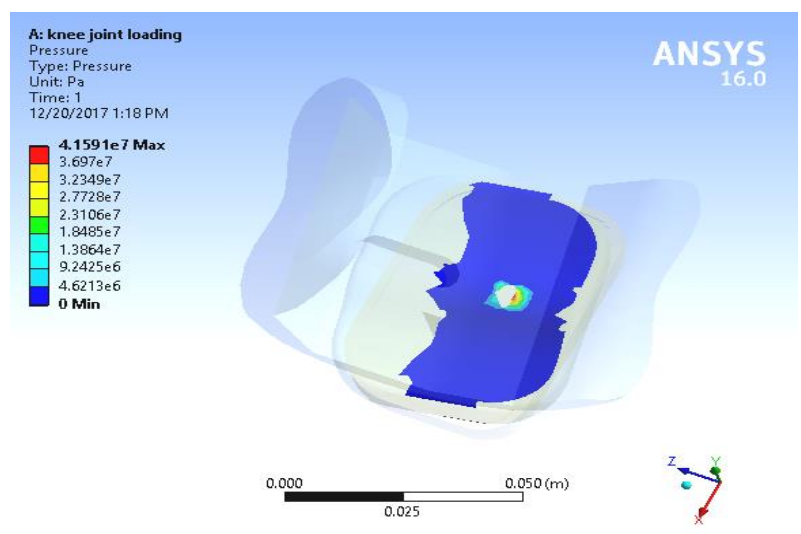

(a) For Co-Cr on UHMWPE Prosthesis

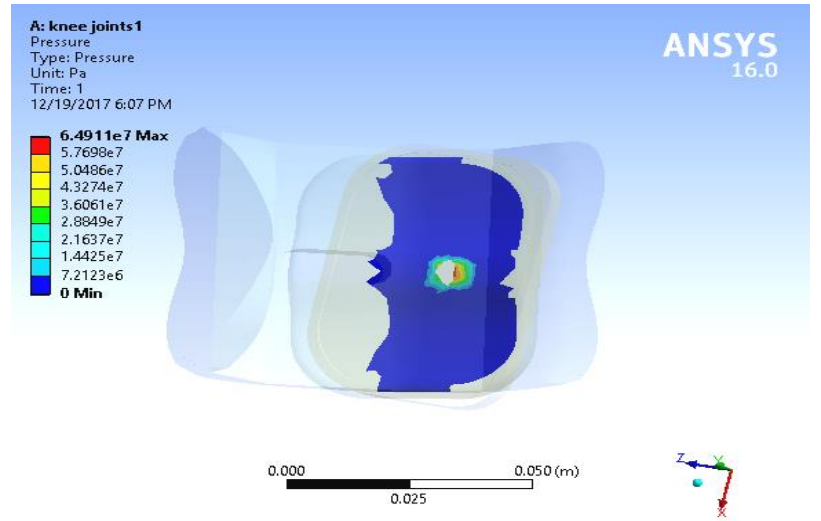

(b) For Co-Cr on PTFE-25 Prosthesis

Figure 9. Contact pressure of knee joint at $1500 \mathrm{~N}$

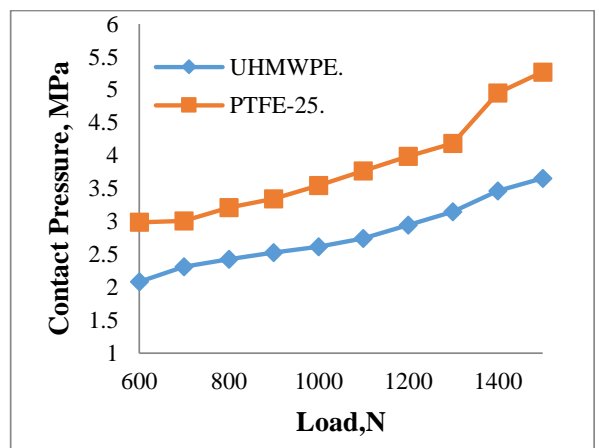

(a) For Hip Joint Prosthesis

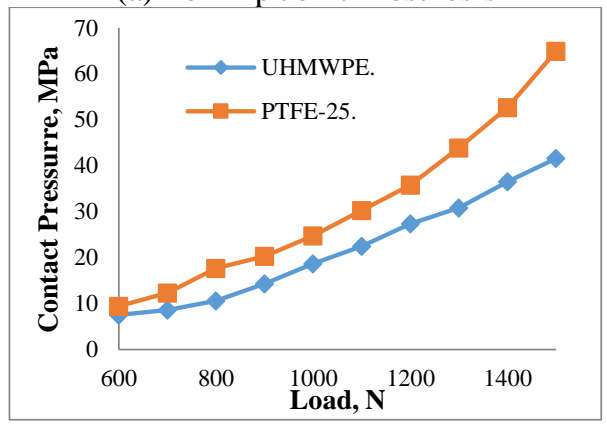

(b) For Knee Joint Prosthesis

Figure 10. Contact pressure vs load

\section{DISCUSSION}

From the Von-mises equivalent stress analysis it has been found that both the hip and knee prostheses using PTFE-25 liners have higher strength than those using UHMWPE under similar loading conditions. Therefore the PTFE-25 plastic acetabular cups for both the prostheses should be preferred over the UHMWPE under identical loading conditions because of higher localized strength. Similarly, the total deformation analysis resulted lower deformation values for PTFE-25 liners than UHMWPE liners for both hip and knee joint prostheses which is highly desirable.

The fine tetrahedron meshing of all the components has led to the proliferation of accurate results in the analysis at all points of applied loads. The variation of load from $600 \mathrm{~N}$ to $1500 \mathrm{~N}$ is applicable for a healthy adult person during simple street walking. Therefore the prostheses should be designed in such a way that it could transmit maximum contact pressure for both hip and knee joints. As we know from Archard's equation that the volumetric wear rate of any two sliding 
surfaces greatly depends upon the contact pressure, sliding distance and the materials wear coefficient. For all cases the values of sliding distances and wear coefficients are much lower than the contact pressures. Therefore, one can assure that the components subjected to higher contact pressure will be subjected to lesser sliding wear. In this case, it is observed that the contact pressures are higher at all points transmitting the loads from $600 \mathrm{~N}$ to $1500 \mathrm{~N}$ for both hip and knee joint prostheses using PTFE-25 as acetabular and tibial liners. So the PTFE-25 should be preferred over the UHMWPE material for the acetabular and distal femur liners of hip and knee joint prostheses.

\section{CONCLUSIONS}

In this study, we developed a finite element model of the hip and knee joint prostheses by simulating the real life conditions with a view to analyze its performances during a normal walking cycle of a human being. It is observed from the above results that the Von-Mises Equivalent stress for all the plastic surfaces sliding against $\mathrm{Co}-\mathrm{Cr}$ surfaces remains same under identical conditions. The Von-mises equivalent stress analysis and total deformation approaches give strength comparison between PTFE-25 and UHMWPE acetabular and tibial liners.

But, the contact pressure analysis from Figures 8(a) and (b) projects very clear comparison between these components. It has been observed from these simulations that the components made of PTFE-25 have greater contact pressure values as compared with that of the UHMWPE for both hip and knee prostheses. This indicates a lower scoring of the contacting surfaces leading to lesser abrasive wear, thereby enhancing the component life. So the novel bio-plastic material PTFE with $25 \%$ glass composite should be preferred over the existing UHMWPE for making the acetabular cups of the Metal-onPlastic hip prostheses for longer life.

Since it is not so easy to conduct a real life experiment for analyzing the performance of a hip prosthesis by frequently using various materials, the computer simulation becomes an alternate choice. Therefore it is strongly recommended to follow the proposed modeling and simulation approach to solve complex problems like stress analysis and wear behaviour of hip and knee joint prostheses before manufacturing the prototype in order to save valuable time and money.

\section{ACKNOWLEDGMENT}

We are thankful to the Director and Head of Mechanical Engineering Department, NIT Agartala, India for their support and motivation to carry out this research work successfully. We are also thankful to the MHRD and AICTE, Govt. of India for their financial support.

\section{REFERENCES}

[1] Keene, G.S., Villar, R.N. (1994). Arthroscopic anatomy of the hip: An in vivo study. Arthroscopy, 10(4): 392-399. https://doi.org/10.1016/S0749-8063(05)80189-6

[2] Schüller, H.M., Marti, R.K. (1990). Ten-year socket wear in 66 hip arthroplasties: Ceramic versus metal heads. Acta Orthop Scand, 61(3): 240-243. https://doi.org/10.3109/174536790089935089

[3] Nine, M., Choudhury, D., Hee, A., Mootanah, R., Osman, N.A.A. (2014). Wear debris characterization and corresponding biological response: Artificial hip and knee joints. Materials, 7(2): 980-1016. http://dx.doi.org/10.3390/ma7020980

[4] Kurtz, S., Ong, K., Lau, E., Mowat, F., Halpern, M. (2007). Projections of primary and revision hip and knee arthroplasty in the United States from 2005 to 2030. J Bone Jt Surg Ser A, 89(4): 780-785. https://doi.org/10.2106/JBJS.F.00222

[5] Rawal, B.R., Yadav, A., Pare, V. (2016). Life estimation of knee joint prosthesis by combined effect of fatigue and wear. Proceedia Technol, 23: 60-67. https://doi.org/10.1016/j.protcy.2016.03.072

[6] Seppänen, M., Karvonen, M., Virolainen, P. (2016). Poor 10-year survivorship of hip resurfacing arthroplasty: 5,098 replacements from the Finnish Arthroplasty Register. Acta Orthop, 87(6): 554-559. https://doi.org/10.1080/17453674.2016.1246316

[7] Roy, T., Choudhury, D., Ghosh, S., Mamat, A.B., Murphy, B.P. (2014). Improved friction and wear performance of micro dimpled ceramic-on-ceramic interface for hip joint Arthroplasty. Ceramics International, S0272-8842(14): $\quad$ 01364-9. http://dx.doi.org/10.1016/j.ceramint.2014.08.123

[8] Espallargas, N., Fischer, A., Igual Muñoz, A., Mischler, S., Wimmer, M.A. (2017). In-situ generated tribomaterial in metal/metal contacts: Current understanding and future implications for implants. Biotribology, 10: 42-50. https://doi.org/10.1016/j.biotri.2017.03.006

[9] Saikko, V. (2009). Friction measurement in the biaxial rocking motion hip joint simulator. J Tribol, 131(1): 011201. https://doi.org/10.1115/1.2991121

[10] Al-Dirini, R.M.A., O’Rourke, D., Huff, D., Martelli, S., Taylor, M. (2018). Biomechanical robustness of a contemporary cementless stem to surgical variation in stem size and position. J. Biomech Eng., 140(9): 091007. https://doi.org/10.1115/1.4039824

[11] Jin, D., Zhang, R., Dimo, H., Wang, R., Zhang, J. (2003). Kinematic and dynamic performance of prosthetic knee joint using six-bar mechanism. J Rehabil Res Dev., 40(1): 39. https://doi.org/10.1682/JRRD.2003.01.0039

[12] Das, S.S., Chakraborti, P. (2018). Development of biomaterial for total hip joint replacement. IOP Conf Ser Mater Sci Eng, 377(1). https://doi.org/10.1088/1757899X/377/1/012177

[13] Paul, M.C., Glennon, L.P., Baer, T.E., Brown, T.D. (2008). Field variable associations with scratch orientation dependence of UHMWPE wear: A finite element analysis. Journal of Biomechanical Engineering, 130(6): 061019. https://doi.org/10.1115/1.2939273 